\title{
Evolution of the Motion Around a Slowly Rotating Body
}

\author{
Z. M. Hayman* and W. M. El-Mahy
}

Astronomy department-Faculty of Science-Cairo University-Egypt

\begin{abstract}
The motion of a satellite about a rotating triaxial body will be investigated, stressing on the case of slow rotation. The Hamiltonian of the problem will be formed including the zonal harmonic $\mathbf{J}_{2}$ and the leading tesseral harmonics $\mathrm{C}_{22}$ and $\mathrm{S}_{22}$. The small parameter of the problem is the spin rate $(\sigma)$ of the primary. The solution proceeds through three canonical transformations to eliminate in succession; the short, intermediate and long-period terms. Thus secular and periodic terms are to be retained up to orders four and two respectively.
\end{abstract}

Keywords: Hamiltonian, perturbation, motion, satellite.

\section{INTRODUCTION}

To understand the dynamics of a spacecraft or a natural particle around a celestial body (a planet, an asteroid or even a comet) it's convenient to take into account the spin rate $(\sigma)$ of the primary, since it is important for several applications especially for geodetic satellites and when dealing with communication satellites where there's commensur-ability between the satellite period and $\sigma$, and a case of resonance arises [1-3]. Considering a slowly rotating earth like planet, the addition of tesseral harmonics is necessary $[4,5]$ analyzed the orbital dynamics about an asteroid and established that the major perturbations acting on the orbiter are due to the leading harmonics of the geopotential. In a subsequent work [6] he tacked the problem of secular motion in a $2^{\text {nd }}$ degree and order-gravity field with no rotation qualitatively. In this respect the main problem of artificial satellite theory is very useful; the major contributors to this subject were [710] formed the Hamiltonian of the motion of an A.S. about a planet with an inhomogeneous gravitational field including the leading zonal and tesseral harmonics. He used the Whittaker variables and then he normalized the Hamiltonian using the method of elimination of the parallax developed by [11].

Instead of obtaining an explicit solution of the problem he performed an exhaustion analysis of the problem. The method adopted by Palacian, through elegant, but is very difficult to include higher order terms and higher order gravity coefficients.

In this paper the gravitational force exerted by an earth like planet on an artificial satellite will be considered, the Hamiltonian of the problem will be formed, in terms of the Delaunay variables, with the earth's spin rate $\sigma$ taken as a small parameter of $\mathrm{O}(1)$. The planet's potential will be considered up to the leading zonal and leading tesseral

*Address correspondence to this author at the Astronomy departmentFaculty of Science-Cairo University-Egypt; Tel: +2010-1001644;

Fax:+202-35717026; E-mails: zmhayman@gmail.com,

zmhayman@yahoo.com harmonics, an outline of the perturbation technique is given which is based on the Lie- Deprit - Kamel transform.

The Hamiltonian is then normalized through three successive canonical transformations to eliminating succession of the short, intermediate, and long period terms. The procedure followed facilitates such including higher order terms and higher coefficients of the geopotential.

\section{THE GEOPOTENTIAL}

The earth's gravitational potential is usually expressed by "Vinti's potential":

$$
\mathrm{V}=-\frac{\mu}{\mathrm{r}} \sum_{\mathrm{n}=0}^{\infty} \sum_{\mathrm{m}=0}^{\mathrm{n}}\left(\frac{\mathrm{R}}{\mathrm{r}}\right)^{\mathrm{n}} \mathrm{P}_{\mathrm{n}}^{\mathrm{m}}(\sin \delta)\left(\mathrm{c}_{\mathrm{nm}} \cos \mathrm{m} \lambda+\mathrm{s}_{\mathrm{nm}} \sin \mathrm{m} \lambda\right)
$$

Where R: the equatorial radius of the earth. $\mu=\mathrm{GM}_{\mathrm{e}}$ is the product of the gravitational constant and the mass of the earth, and known as the earth's gravitational parameter, $(\mathrm{r}, \lambda$, $\delta)$ are the geocentric coordinates with $\lambda$ being measured east of Greenwich, $\mathrm{P}_{\mathrm{n}}^{\mathrm{m}}(\sin \delta)$ are the associated legender polynomials, and $c_{n m}$ and $s_{n m}$ are harmonic coefficients. The terms with $\mathrm{m}=0$ correspond to zonal harmonics, those with $0<\mathrm{m}<\mathrm{n}$ correspond to tesseral harmonics, while $\mathrm{m}=\mathrm{n}$ correspond to sectorial harmonics, $\mathrm{J}_{2}$ measures the equatorial bulge of the earth and $\mathrm{C}_{22}, \mathrm{~S}_{22}$ measure the elliptical shape of the earth's equator. The coefficients $\mathrm{C}_{21}$ and $\mathrm{S}_{21}$ are vanishing small and since the origin is taken at the center of mass, the coefficients $\mathrm{C}_{10}, \mathrm{C}_{11}$ and $\mathrm{S}_{11}$ will be zero; also both the tesseral and sectorial harmonics will be simply referred to as tesseral harmonics. With the previous considerations, and writing the zonal and tesseral harmonics separately, eqn. (1) will be:

$$
\mathrm{v}=-\frac{\mu}{\mathrm{r}}+\sum_{\mathrm{n}=2}^{\infty} \mathrm{Z}_{\mathrm{n}}^{\prime}+\sum_{\mathrm{n}=2}^{\infty} \sum_{\mathrm{m}=2}^{\mathrm{n}} \mathrm{T}_{\mathrm{nm}}^{\prime}
$$


Where $\mathrm{Z}_{\mathrm{n}}^{\prime}=\mu \mathrm{R}^{\mathrm{n}} \mathrm{J}_{\mathrm{n}} \frac{\mathrm{P}_{\mathrm{n}}(\sin \delta)}{\mathrm{r}^{\mathrm{n}+1}}, \mathrm{~J}_{\mathrm{n}}=-\mathrm{c}_{\mathrm{n} 0}$

$\mathrm{T}_{\mathrm{nm}}^{\prime}=-\mu \mathrm{R}^{\mathrm{n}}\left(\mathrm{C}_{\mathrm{nm}} \cos \mathrm{m} \lambda+\mathrm{S}_{\mathrm{nm}} \sin \mathrm{m} \lambda\right) \frac{\mathrm{P}_{\mathrm{n}}^{\mathrm{m}}(\sin \delta)}{\mathrm{r}^{\mathrm{n}+1}}$

Taking into account the orders considered only and using the associated legendre functions and the legender polynomial formulas then:

$\left.\mathrm{P}_{2}=\frac{1}{4}\left[\left(3 \mathrm{~S}^{2}-2\right)-3 \mathrm{~S}^{2} \cos \mathrm{F}_{22}\right)\right]$

$\mathrm{P}_{2}^{2}(\sin \delta)=3 \cos ^{2} \delta$

Setting if $+j \omega=F_{i j}, C=\cos I, S=\sin I$

And substituting $\sin \delta=\mathrm{S} \sin \mathrm{F}_{11}$

Where $\mathrm{I}$ is the orbital inclination, $\mathrm{f}$ is the true anomaly and $\omega$ is the argument of perigee, then using the trigonometric formulae developed by [12-15] the factors $\left(\cos ^{\mathrm{m}} \delta\right)$ can be cancelled out. If $\Omega$ is the longitude of the node measured east ward from the (rotating) meridian of Greenwich, then from the Fig* (1).

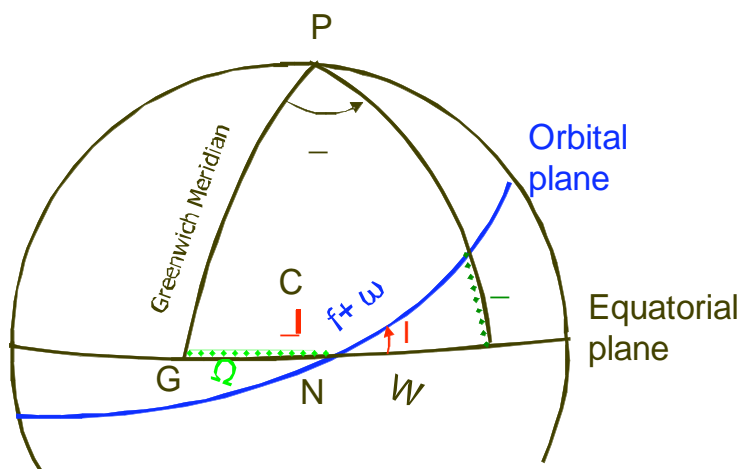

Fig. (1). Celestial Sphere (1).

$\lambda=\Omega+W$

$\cos W=\frac{\cos F_{11}}{\cos \delta}, \sin W=\frac{C \sin F_{11}}{\cos \delta}$

Setting $\mathrm{C}_{1}=\cos \mathrm{F}_{11}, \mathrm{~S}_{1}=\mathrm{C} \sin \mathrm{F}_{11}$

$\sin \lambda=\frac{C_{1} \sin \Omega+S_{1} \cos \Omega}{\cos \delta}$

$\cos \lambda=\frac{C_{1} \cos \Omega-S_{1} \sin \Omega}{\cos \delta}$

From (10) and using De Moivre's theorem

$$
\begin{aligned}
& \cos m \lambda+i \sin m \lambda=(\cos \lambda+i \sin \lambda)^{m} \\
& =\frac{1}{\cos ^{m} \delta}(\cos m \Omega+i \sin m \Omega)\left(C_{1}+i S_{1}\right)^{m} \\
& =\frac{1}{\cos ^{m} \delta}(\cos m \Omega+i \sin m \Omega) \sum_{r=0}^{m}\left(\begin{array}{l}
m \\
r
\end{array}\right)\left(i S_{1}\right)^{r} C_{1}^{m-r}
\end{aligned}
$$

Equating the real and imaginary parts, we get

$$
\begin{aligned}
\sin m \lambda & =\frac{C_{m} \sin m \Omega+S_{m} \cos m \Omega}{\cos ^{m} \delta} \\
\cos m \lambda & =\frac{C_{m} \cos m \Omega-S_{m} \sin m \Omega}{\cos ^{m} \delta}
\end{aligned}
$$

Where

$$
\begin{aligned}
& C_{m}=\sum_{j \geq 0}^{m}\left(\begin{array}{c}
m \\
2 j
\end{array}\right)\left(i S_{1}\right)^{2 j} C_{1}^{m-2 j} \\
& S_{m}=\frac{1}{i} \sum_{j \geq 0}^{m}\left(\begin{array}{c}
m \\
2 j+1
\end{array}\right)\left(i S_{1}\right)^{2 j+1} C_{1}^{m-2 j-1}
\end{aligned}
$$

Then from (12) we get $\begin{aligned} & C_{2}=C_{1} C_{1}-S_{1} S_{1} \\ & S_{2}=S_{1} C_{1}-C_{1} S_{1}\end{aligned}$

The general relation, which can be generalized to the recursive relation so from $\left(9^{*}, 13\right)$ we get

$C_{2}=\frac{S_{2}}{2}+\left(1-\frac{S_{2}}{2}\right) \cos F_{22}, S_{2}=C \sin F_{22}$

And the tesseral harmonics become in the form

$T_{n m}^{\prime}=\frac{-\mu R^{n}}{r^{n+1}} \frac{P_{n}^{m}(\sin \delta)}{\cos ^{m} \delta}\left[\left(C_{n m} \cos m \Omega+S_{n m} \sin m \Omega\right) C_{m}+\left(S_{n m} \cos m \Omega-C_{n m}\right.\right.$ $\left.\sin m \Omega) S_{m}\right]$

\section{THE HAMILTONIAN IN TERMS OF THE DELAUNAY VARIABLES}

If we consider the Delaunay set of canonical variables

$$
\begin{aligned}
& \mathrm{L}_{\mathrm{D}}=\sqrt{\mu \mathrm{a}}, l_{D}=M_{\text {mean }} \text { (Mean anomaly) } \\
& G_{D}=L_{D} \sqrt{1-e^{2}}, g_{D}=\omega \text { (argument of periapsis) } \\
& H_{D}=C G_{D}, h_{D}=\Omega \text { (Longitude of the node) }
\end{aligned}
$$

Then the equations of motion become

$\dot{y}_{D}=M\left(\frac{\partial \hat{\mathrm{H}}}{\partial y_{D}}\right)^{T}, \mathrm{y}_{\mathrm{D}}=\operatorname{col}\left(\mathrm{l}_{\mathrm{D}}, \ldots, \mathrm{H}_{\mathrm{D}}\right)$

Where $M$ is the canonical matrix $M=\left(\begin{array}{cc}0 & I_{n} \\ -I_{n} & 0\end{array}\right), I$ is the identity matrix. And $\hat{\mathrm{H}}$ is expressed in terms of the above set of canonical variables. $\hat{H}$ depends explicitly on time due to the measurement of $h_{D}$ from the rotating meridian of Greenwich. The autonomous form of the equations is recovered by:

(i). Adjoining to the above set a new pair of conjugate variables $\left(\mathrm{k}_{\mathrm{D}}, \mathrm{K}_{\mathrm{D}}\right)$ where $\mathrm{k}_{\mathrm{D}}=v \mathrm{t}+$ const., and augmenting the Hamiltonian such that: 


$$
\dot{k}_{D}=v=\frac{\partial \mathrm{H}_{D}}{\partial K_{D}}, \dot{K}_{D}=-\frac{\partial \mathrm{H}_{D}}{\partial k_{D}}
$$

Since $\hat{\mathrm{H}}$ does not depend on $K_{D}$, it can be taken as constant of integration. So that:

$\mathrm{H}_{D}=v K_{D}+\hat{\mathrm{H}}$

The variable $K_{D}$ may be identified by means of the second of equations (19).

(ii). Performing a canonical transformation so that the new angular variables become:

$l=l_{D}, g=g_{D}, h=h_{D}-\sigma t, k=k_{D}$

Where $\sigma$ is the angular speed of the earth. To find the new momenta and the new Hamiltonian, we have:

$$
\begin{aligned}
& \left(L_{D} d l+G_{D} d g+H_{D} d h_{D}+K_{D} d k\right)- \\
& {\left[L d l+G d g+H\left(d h_{D}-\sigma d t\right)+K d t\right]+\left(\mathrm{H}-\mathrm{H}_{D}\right) d t=d \tau}
\end{aligned}
$$

Where $d \tau$ is the total differential of a function that we choose to be zero. Equating the coefficients of earth differential to zero yields:

$L=L_{D}, G=G_{D}, H=H_{D}, K=K_{D}$

And $\mathrm{H}=\mathrm{H}_{D}-\sigma H$

So in terms of this set of variables the equations of motion become:

$$
\dot{z}=M \mathrm{H}_{z}^{T} \text { Where } \mathrm{z}=\operatorname{col}\left(\mathrm{l}_{\mathrm{D}}, \ldots, \mathrm{K}_{\mathrm{D}}\right)
$$

and $\mathrm{H}=\frac{-\mu^{2}}{2 L^{2}}-\sigma H+\sum_{n}^{2} Z_{n}^{\prime}+\sum_{n}^{2} \sum_{m}^{n} T_{n m}^{\prime}$

Considering $\sigma$ as the small parameter of the problem, the orders of magnitude of the involved parameters are defined as:

$$
\sigma \equiv o(1), J_{2} \equiv o(2), C_{n m}, S_{n m} \equiv o(4)
$$
of $\sigma$

The Hamiltonian can now be expressed as a power series

$$
\mathrm{H}=\sum_{n=0}^{4} \frac{\sigma^{n}}{n !} \mathrm{H}_{n}
$$

Where we retain the component of (28) up to $4^{\text {th }}$ order in $\sigma$, i.e. up to $\mathrm{J}_{2}$ in zonal harmonics and up to $\mathrm{C}_{22}, \mathrm{~S}_{22}$ in tesseral harmonics, so the components will be as follows:

$$
\begin{aligned}
& \mathrm{H}_{0}=\frac{-\mu^{2}}{2 L^{2}} \\
& \mathrm{H}_{1}=-H
\end{aligned}
$$

$$
\mathrm{H}_{2}=\frac{A_{2}}{L^{6}} \varphi^{3} z_{2}
$$

$\mathrm{H}_{3}=0$

$$
\mathrm{H}_{4}=\frac{\varphi^{3}}{L^{6}} T_{22}
$$

Where $A_{2}$ is a zero order constant, according to equations (26), (3) and (5) is given by:

$$
\begin{aligned}
& A_{2}=\frac{\mu^{4} R^{2} J_{2}}{2 \sigma^{2}} \\
& z_{2}=\left(3 S^{2}-2\right)-3 S^{2} \cos F_{22}
\end{aligned}
$$

And $T_{21}=0 \quad \mathrm{C}_{21}, \mathrm{~S}_{21} \rightarrow 0$

$T_{22}=\gamma_{22}\left[S^{2}+\left(2-S^{2}\right) \cos F_{22}\right]+2 \Gamma_{22} C \sin F_{22}$

Where $\gamma_{22}=A_{22} \cos 2 h+B_{22} \sin 2 h$

$\Gamma_{22}=B_{22} \cos 2 h-A_{22} \sin 2 h$

And the $\mathrm{A}_{22}, \mathrm{~B}_{22}$ are zero order constants given by:

$$
\begin{aligned}
& A_{22}=\frac{-36 \mu^{4} R^{2}}{\sigma^{4}} c_{22} \\
& B_{22}=\frac{-36 \mu^{4} R^{2}}{\sigma^{4}} s_{22}
\end{aligned}
$$

\section{THE PERTURBATION TECHNIQUE}

We now outline the perturbation technique up to $4^{\text {th }}$ order in the secular, $3^{\text {rd }}$ order in the intermediate and $2^{\text {nd }}$ order in the short periodic terms.

Let $\varepsilon$ be the small parameter of the problem and let the considered system of differential equations to be:

$$
\dot{u}=\mathrm{H}_{U}^{T}, \dot{U}=-\mathrm{H}_{u}^{T}
$$

Where $(\mathrm{u}, \mathrm{U})$ is the six-vector of adopted canonical variables, the Hamiltonian is assumed expandable as:

$$
\mathrm{H}=\mathrm{H}_{0}+\sum \frac{\varepsilon^{n}}{n !} \mathrm{H}_{n}
$$

And the system with $\mathrm{H}=\mathrm{H}_{0}$ is assumed integrable with $\mathrm{H}_{0}=\mathrm{H}_{0}\left(U_{1}\right)$

What is required is to construct three canonical transformation $(\mathrm{u}, \mathrm{U} ; \varepsilon) \rightarrow\left(\mathrm{u}^{\prime}, \mathrm{U}^{\prime}\right), \quad\left(\mathrm{u}^{\prime}, \mathrm{U}^{\prime} ; \varepsilon\right) \rightarrow\left(\mathrm{u}^{\prime \prime}, \mathrm{U}^{\prime \prime}\right) \quad$ and $\left(\mathrm{u}^{\prime \prime}, \mathrm{U}^{\prime \prime} ; \varepsilon\right) \rightarrow\left(\mathrm{u}^{\prime \prime \prime}, \mathrm{U}^{\prime \prime \prime}\right)$ analytic in $\varepsilon$ at $\varepsilon=0$ to eliminate in succession the short, intermediate and long period terms from the Hamiltonian such that $\mathrm{U}^{\prime \prime \prime}$ reduce to constants and $u^{\prime \prime \prime}$ become linear functions of time, where the short period terms are those periodic in the mean anomaly, $\mathrm{u}_{1}=1$, the 
intermediate terms are those periodic in the longitude of the node, $\mathrm{u}_{2}=\mathrm{h}$, and the long period terms are those periodic in the argument of perigee, $\mathrm{u}_{3}=\mathrm{g}$. The transformated Hamiltonians and the corresponding generators will be assumed expandable as:

$$
\begin{aligned}
& \mathrm{H}^{*}\left(-, u_{2}^{\prime}, u_{3}^{\prime} ; U^{\prime} ; \varepsilon\right)=\mathrm{H}_{0}^{*}\left(U_{1}^{\prime}\right)+\sum_{n=1} \frac{\varepsilon^{n}}{n !} \mathrm{H}_{n}^{*}\left(-, u_{2}^{\prime}, u_{3}^{\prime} ; U^{\prime}\right) \\
& \mathrm{H}^{* *}\left(-, u_{3}^{\prime \prime} ; U^{\prime \prime} ; \varepsilon\right)=\mathrm{H}_{0}^{* *}\left(U_{1}^{\prime \prime}\right)+\sum_{n=1} \frac{\varepsilon^{n}}{n !} \mathrm{H}_{n}^{* *}\left(-, u_{3}^{\prime \prime} ; U^{\prime \prime}\right) \\
& \mathrm{H}^{* * * *}\left(-; U^{\prime \prime \prime} ; \varepsilon\right)=\mathrm{H}_{0}^{* * *}\left(U_{1}^{\prime \prime \prime}\right)+\sum_{n=1} \frac{\varepsilon^{n}}{n !} \mathrm{H}_{n}^{* * *}\left(-; U^{\prime \prime \prime}\right) \\
& w\left(u^{\prime}, U^{\prime} ; \varepsilon\right)=\sum_{n=0} \frac{\varepsilon^{n}}{n !} w_{n+1}\left(u^{\prime}, U^{\prime}\right) \\
& w^{*}\left(-, u_{2}^{\prime \prime}, U^{\prime \prime} ; \varepsilon\right)=\sum_{n=0} \frac{\varepsilon^{n}}{n !} w^{*}{ }_{n+1}^{*}\left(-, u_{2}^{\prime \prime}, U^{\prime \prime}\right) \\
& w^{* *}\left(-, u_{3}^{\prime \prime \prime}, U^{\prime \prime \prime} ; \varepsilon\right)=\sum_{n=0} \frac{\varepsilon^{n}}{n !} w^{* *}\left(-, u_{3}^{\prime \prime \prime}, U^{\prime \prime \prime}\right)
\end{aligned}
$$

We will make use of the following equations during the process of elimination.

$$
\begin{aligned}
& G_{j}=L_{j}-\sum_{m=0}^{j-2}\left(\begin{array}{c}
j-1 \\
m
\end{array}\right) L_{m+1} G_{j-m-1} \\
& \mathrm{H}_{0}^{*}=\mathrm{H}_{0} \\
& \mathrm{H}_{n}^{*}=\mathrm{H}_{n}+L_{n} \mathrm{H}_{0}+\sum_{j=1}^{n-1}\left[\left(\begin{array}{c}
n-1 \\
j-1
\end{array}\right) L_{j} \mathrm{H}_{n-j}+\left(\begin{array}{c}
n-1 \\
j
\end{array}\right) G_{j} \mathrm{H}_{n-j}^{*}\right], n \geq 1
\end{aligned}
$$

\section{ELIMINATION OF SHORT PERIOD TERMS}

The basic identities are:

$$
\left.\begin{array}{l}
\mathrm{H}_{0}^{*}=\mathrm{H}_{0} \\
\mathrm{H}_{n}^{*}=\tilde{\mathrm{H}}_{n}+\left(\mathrm{H}_{0} ; w_{n}\right) \\
\tilde{\mathrm{H}}_{n}=\mathrm{H}_{n}+\sum_{j=1}^{n-1}\left\{\left(\begin{array}{c}
n-1 \\
j-1
\end{array}\right)\left(\mathrm{H}_{n-j} ; w_{j}\right)+\left(\begin{array}{c}
n-1 \\
j
\end{array}\right) G_{j} \mathrm{H}_{n-j}^{*}\right.
\end{array}\right\}
$$

Let $u_{1}^{\prime}$ be the fast variable in $\mathrm{H}$, we choose $\mathrm{H}_{n}^{*}$ to be the average of $\tilde{H}_{n}$ over $u_{1}^{\prime}$; i.e.

$$
\begin{aligned}
& \mathrm{H}_{n}^{*}=\left\langle\tilde{\mathrm{H}}_{n}\right\rangle_{u_{1}^{\prime}} \\
& P_{n}=\tilde{\mathrm{H}}_{n}-\mathrm{H}_{n}^{*}=\left(w_{n} ; \mathrm{H}_{0}\right) \\
& \text { So that: } w_{n}=\left(\frac{\partial \mathrm{H}_{0}}{\partial U_{1}^{\prime}}\right)^{-1} \int P_{n} d u_{1}^{\prime}
\end{aligned}
$$

Where we apply the previous equations up to $\mathrm{H}_{4}^{*}, w_{2}$.

\section{ELEMENTS OF THE SHORT PERIOD TRANS- FORMATION AND ITS INVERSE}

These are obtained from the equations for the vector transformation, namely:

$$
\begin{aligned}
& u=u^{\prime}+\sum_{n=1}^{3} \frac{\varepsilon^{n}}{n !} u^{\prime(n)} \\
& U=U^{\prime}+\sum_{n=1}^{3} \frac{\varepsilon^{n}}{n !} U^{\prime(n)}
\end{aligned}
$$

Where

$$
\begin{aligned}
& u^{\prime(n)}=\frac{\partial w_{n}}{\partial U^{\prime}}+\sum_{j=1}^{n-1}\left(\begin{array}{c}
n-1 \\
j
\end{array}\right) G_{j} u^{\prime(n-j)}, n \geq 1 \\
& U^{\prime(n)}=-\frac{\partial w_{n}}{\partial u^{\prime}}+\sum_{j=1}^{n-1}\left(\begin{array}{c}
n-1 \\
j
\end{array}\right) G_{j} U^{(n-j)}, n \geq 1
\end{aligned}
$$

And for the inverse transformation:

$$
\begin{aligned}
& u^{\prime}=u+\sum_{n=1}^{3} \frac{\varepsilon^{n}}{n !} u^{(n)}(u, U) \\
& U^{\prime}=U+\sum_{n=1}^{3} \frac{\varepsilon^{n}}{n !} U^{(n)}(u, U)
\end{aligned}
$$

Where

$$
\begin{aligned}
& u^{(n)}=-u^{\prime(n)}+\sum_{j=1}^{n-1}\left(\begin{array}{l}
n \\
j
\end{array}\right) G_{j} u^{\prime(n-j)}, n \geq 1 \\
& U^{(n)}=-U^{\prime(n)}+\sum_{j=1}^{n-1}\left(\begin{array}{l}
n \\
j
\end{array}\right) G_{j} U^{\prime(n-j)}, n
\end{aligned}
$$

\section{THE INTERMEDIATE TRANSFORMATION}

The procedure is essentially similar to that at the short period transformation with the averages taken over $u_{2}^{\prime \prime}$, so:

$$
\begin{aligned}
& \mathbf{H}_{0}^{* * *}=\mathbf{H}_{0}^{*} \\
& \mathbf{H}_{n}^{* * *}=\mathbf{H}_{n}^{*}+\sum_{j=1}^{n-1}\left\{\left(\begin{array}{c}
n-1 \\
j-1
\end{array}\right)\left(\mathbf{H}_{n-j}^{*} ; w_{j}^{*}\right)+\left(\begin{array}{c}
n-1 \\
j
\end{array}\right) G_{j}^{*} \mathbf{H}_{n-j}^{* *}\right\}+\left(\mathbf{H}_{0}^{*} ; w_{n}^{*}\right),
\end{aligned}
$$

Since $\mathrm{H}_{n}^{* *}$ and $\mathrm{H}_{n}^{*}$ are independent of $u_{2}^{\prime \prime}$, we may choose $w_{n}^{*}$ also independent of $u_{1}^{\prime \prime}$, then the last term vanishes and the last equation reduces to

$$
\mathrm{H}_{n}^{* *}=\mathrm{H}_{n}^{*}+\sum_{j=1}^{n-1}\left\{\left(\begin{array}{c}
n-1 \\
j-1
\end{array}\right)\left(\mathrm{H}_{n-j}^{*} ; w_{j}^{*}\right)+\left(\begin{array}{c}
n-1 \\
j
\end{array}\right) G_{j}^{*} \mathrm{H}_{n-j}^{* *}\right\}
$$

The equations involved may be out lined as before with the change $\mathrm{H} \rightarrow \mathrm{H}^{*}, \mathrm{H}^{*} \rightarrow \mathrm{H}^{* *}, w \rightarrow w^{*}, u^{\prime}, U^{\prime} \rightarrow u^{\prime \prime}, U^{\prime \prime}$

Order (1) 


$$
\mathrm{H}_{1}^{* *}=\mathrm{H}_{1}^{*}+\left(\mathrm{H}_{0}^{*} ; w_{1}^{*}\right)=\mathrm{H}_{1}^{*}
$$

Where we note that $\mathrm{H}_{0\left(U_{1}^{\prime}=U_{1}^{\prime \prime}\right)}^{*}=\mathrm{H}_{0}^{*}\left(U^{\prime \prime}\right)$ and $w^{*}$ is independent at $u_{1}^{\prime \prime}$ so $\left(\mathrm{H}_{0}^{*} ; w_{1}^{*}\right)=0$

Order (2)

$$
\begin{aligned}
& G_{1}=L_{1}^{*} \\
& \mathrm{H}_{2}^{* *}=\mathrm{H}_{2}^{*}+\left(\mathrm{H}_{1}^{*} ; w_{1}^{*}\right)+\left(\mathrm{H}_{1}^{* *} ; w_{1}^{*}\right)=\mathrm{H}_{2}^{*}+2\left(\mathrm{H}_{1}^{*} ; w_{1}^{*}\right)
\end{aligned}
$$

We choose

$$
\begin{aligned}
& \mathrm{H}_{2}^{* *}=\left\langle\mathrm{H}_{2}^{*}\right\rangle_{u_{2}^{\prime \prime}} \\
& P_{2}^{*}=\mathrm{H}_{2}^{*}-\mathrm{H}_{2}^{* *}=2\left(w_{1}^{*} ; \mathrm{H}_{1}^{*}\right)
\end{aligned}
$$

Let

$$
P_{n}^{*}=\sum_{i, j} c_{n}^{i j} \cos \left(i u_{2}^{\prime \prime}+j u_{3}^{\prime \prime}+\alpha_{n}^{i j}\right)
$$

Where $c_{n}$ are functions of $\mathrm{U}_{1}^{\prime \prime}$ and $\alpha_{n}^{i j}$ are numerical constants to account for the phase then

$$
w_{1}^{*}=\frac{1}{2} \sum_{i, j} \frac{c_{2}^{i j} \sin \left(i u_{2}^{\prime \prime}+j u_{3}^{\prime \prime}+\alpha_{2}^{i j}\right)}{i \frac{\partial \mathrm{H}_{1}^{*}}{\partial u_{2}^{\prime \prime}}+j \frac{\partial \mathrm{H}_{1}^{*}}{\partial u_{3}^{\prime \prime}}}
$$

Similarly we find order (3), elements of the intermediate transformation are obtained by the same equations used for the short period transformation and the use of the interchanges used previously. The long period transformation and its elements are found in a similar manner.

\section{NORMALIZATION}

Equation (28) shows that the Hamiltonian of the problem is degenerate with $l$ is the fast variable, $h$ is intermediate variable, and $g$ is slow one.

So the Hamiltonian (28) is normalized through three successive canonical transformations.

\subsection{The Short Period Terms}

Averaging over $l$ and following the steps outlined in the procedure for the short period transformation we obtain

$$
\begin{aligned}
& \mathrm{H}_{0}=-\frac{\mu^{2}}{2 L^{2}} \\
& \mathrm{H}_{1}^{*}=-H^{\prime} \\
& \mathrm{H}_{2}^{*}=\frac{A_{2}}{L^{\prime 6}}\left\langle\phi^{3} z_{2}\right\rangle_{l^{\prime}}=A_{2} \frac{\eta^{\prime 3}}{L^{\prime 6}}\left(3 s^{\prime 2}-2\right) \\
& \mathrm{H}_{3}^{*}=0
\end{aligned}
$$

$\mathrm{H}_{4}^{*}=\tilde{T}_{22}^{\prime}+\frac{9 A_{2}^{2}}{\mu^{2}} \sum_{i=0}^{2}\left(\theta_{i}^{c} \cos 2 i g+\theta_{i}^{s} \sin 2 i g\right)$

$w_{1}=0$

$w_{2}=\frac{A_{2}}{\mu^{2} G^{\prime 3}}\left\{\left(3 s^{\prime 2}-2\right)\left(f^{\prime}-l^{\prime}+e^{\prime} \sin f^{\prime}\right)-\frac{3}{2} s^{\prime 2}\left[\frac{e}{3} \sin F_{32}+\sin F_{22}+e \sin F_{12}\right]\right\}$

8.2. Averaging over $h^{\prime \prime}$ and Following the Procedure Illustrated we Get

$$
\begin{aligned}
& \mathrm{H}_{0}^{* *}=\mathrm{H}_{0}^{*}=-\frac{\mu^{2}}{2 L^{\prime 2}} \\
& \mathrm{H}_{1}^{* *}=\mathrm{H}_{1}^{*}=-H^{\prime \prime} \\
& \mathrm{H}_{2}^{* *}=\mathrm{H}_{2}^{*}=A_{2} \eta_{33}^{\prime \prime} \theta_{33}^{\prime \prime} \\
& \mathrm{H}_{3}^{* *}=0 \\
& \mathrm{H}_{4}^{* *}=\frac{9 A_{2}^{2}}{\mu^{2}} \sum_{0}^{2}\left(\theta_{i}^{c^{\prime \prime}} \cos 2 i g^{\prime \prime}+\theta_{i}^{s^{\prime \prime}} \sin 2 i g^{\prime \prime}\right)
\end{aligned}
$$

$w_{1}^{*}=0$

$w_{2}^{*}=0$

\subsection{Long Period Transformation}

Averaging over $g^{\prime \prime \prime}$ and following the procedure we get

$$
\begin{aligned}
& \mathrm{H}_{0}^{* * * * *}=-\frac{\mu^{2}}{2 L^{\prime \prime 2}} \\
& \mathbf{H}_{1}^{* * * * *}=-H^{\prime \prime \prime} \\
& \mathrm{H}_{2}^{* * * * *}=A_{2} \eta_{33}^{\prime \prime \prime} \theta_{33}^{\prime \prime \prime} \\
& \mathrm{H}_{3}^{* * * * *}=0 \\
& \mathrm{H}_{4}^{* * * *}=\frac{9 A_{2}^{2}}{\mu^{2}} \theta_{0}^{c^{\prime \prime}} \\
& w_{1}^{* * *}=0 \\
& w_{2}^{* * *}=\frac{1}{6 I_{c}^{\prime \prime \prime}}\left[\frac{9 A_{2}^{2}}{2 \mu^{2}} \sum_{i=1}^{2} \frac{1}{i}\left(\theta_{i}^{c^{\prime \prime}} \sin 2 i g^{\prime \prime}-\theta_{i}^{s^{\prime \prime}} \cos 2 i g^{\prime \prime}\right)\right]
\end{aligned}
$$

\section{CONCLUSION}

The present work aims at formulating the problem of the motion of an artificial satellite around a slowly rotating planet, this problem has many applications particularly for missions launched to study the potential harmonics of the earth(or any other planet),and for communication satellites. The formulation is developed in a simple canonical form expressed in terms of a set of Delaunay elements modified to allow for the appearance of the independent variable in the Hamiltonian, this enables using very powerful tools for solv- 
ing for the motion using canonical perturbation approaches such as the methods of von Ziepel's and those based on a Lie series and transform. It is important to stress the importance of such formulation to any lunar satellite (due to the slow rotation of the moon). Though the solution would require some modification due to the varying nature of the lunar potential. In similar works $\mathbf{J}_{2}$ is usually used as the small parameter which provides no simple means to account for the rotation speed either slow or fast. In the present work the rotation speed $\sigma$ is used as the small parameter, thus enabling to account for its order.

\section{ACKNOWLEDGEMENT OF FUNDING}

None declared.

\section{CONFLICTS OF INTEREST}

None declared.

\section{REFERENCES}

[1] Harris AW. Tumbling asteroids. Icarus 1994; 107: 209-11

[2] Hudson RS, Ostro SJ. Shape and non-principle axis spin state of asteroids toutatis. Science 1995; 270: 84-6.
[3] Pravec P, Harris AW. Fast and slowly rotating asteroids. Icarus 2000; 148(1): 12-20.

[4] Scheeres DJ. Dynamics about uniformly rotating triaxial ellipsoids. Icarus 1994; 110: 225-38.

[5] Scheeres DJ, Ostro SJ, Hunson RS, DeJong EM, Suzuki S Dynamics of Orbits Close to Asteroid 4179 Toutatis. Icarus 1998; 132(1): 53-79.

[6] Scheeres DJ, Hu W. Secular Motion in $2^{\text {nd }}$ Degree and OrderGravity Field with no Rotation, Celest Mech Dyn Astr 2001; 79: 183-200.

[7] Brouwer D. Solution of the problem of artificial satellite theory without drag. Astron J 1959; 64: 378-97.

[8] Kozai Y. The motion of a close Earth satellite. Astron J 1959; 64 : 367.

[9] Brouwer D, Clemence GM. Methods of celestial mechanics. New York: Academic Press inc 1961

[10] Palacian JF. Dynamics of a satellite orbiting a planet with an inhomogeneous gravitational field. Celest Mech Dyn Astr 2007; 98: 219-49.

[11] Deprit A. The elimination of the parallax in satellite theory. Celest Mech 1981; 24: 111-53.

[12] Garfinkel B. The Disturbing Function for an Artificial Satellite. Astron J 1965; 70: 688.

[13] Kamal M. Artificial satellite theory including the gravitational effects up to $S_{55}$. Egypt: Cairo University 1982.

[14] Deprit A. Canonical transformations depending on a small parameter. Celest Mech 1968; 1: 12-30.

[15] Kamel AA. Expansion formulae in canonical transformations depending on a small parameter. USA: Stanford University 1969.

Received: December 12, 2011

() Hayman and El-Mahy; Licensee Bentham Open.

This is an open access article licensed under the terms of the Creative Commons Attribution Non-Commercial License (http://creativecommons.org/licenses/by-nc/3.0/) which permits unrestricted, non-commercial use, distribution and reproduction in any medium, provided the work is properly cited. 